\title{
Orientation relative to the retina determines perceptual organization
}

\author{
BARBARA GILLAM and DANIEL McGRATH \\ State University of New York, College of Optometry, 100 East 24th Street, New York, New York 10010
}

\begin{abstract}
Two experiments are reported which show that perceptual unit formation, as measured by Gillam's method of using common reversals of ambiguous lines as an index of their coherence, depends strongly on the orientation of the configuration. Measurements under conditions of $90^{\circ}$ head and body tilt show that the critical orientation is retinal, not physical. The results are discussed in relation to other findings and to possible explanations.
\end{abstract}

The studies to be reported are a continuation of a series using a new criterion and method of measuring perceptual unit formation or grouping. By these terms (which we use interchangeably), we mean the process by which visual input is organized or aggregated into objects or surfaces. This process must precede recognition, although it could also be influenced by it. Grouping must also provide the means by which redundancies are taken advantage of in resolving depth and motion. Indeed, certain depth cues, such as linear perspective, are extracted from relationships among elements in an array, which means they depend upon organization for their existence.

So far, the elements whose grouping we have investigated have been two straight, usually oblique line segments rotated around a central vertical axis (see Figure 1A). The subject viewed a parallel projection of the rotating lines achieved either by means of a shadow caster with a collimating lens (Gillam, 1972) or a computer-controlled CRT display. So long as the projection of each rotating oblique line changes its orientation and length, which it does in a parallel projection, a powerful kinetic depth effect (Wallach \& O'Connell, 1953) is obtained. There is, however, no information as to direction of rotation (clockwise or counterclockwise) in a parallel projection. In the face of this ambiguity, reversals of apparent motion are frequent. The critical issue for our purpose is the degree to which resolutions of this ambiguity are arrived at independently (as opposed to jointly) for two or more lines. The proportion of time that lines appear to rotate in opposite directions can be measured and used as an index of this

This work was supported by Grant B043254 from the National Science Foundation and Grant 30840 from the National Institute of Mental Health. We wish to thank Harold Sedgwick for commenting on the manuscript. D. McGrath is now at the Department of Psychology, University of Lowell, Lowell, Massachusetts 01854. independence (Gillam, 1972). For a strongly grouped configuration of lines, such as a Necker cube or Ames window, reversals would always occur for all lines together. However, grouping is not so perfect for discrete lines and reports of apparent rotation of such lines in opposite directions from one another are frequent. A number of parameters of two lines and their relationship (configurational factors) have been shown to have a strong influence on the degree of grouping obtained. Among these factors are the relative orientation of the lines (Gillam, 1972), their separation (Gillam, 1972), what is placed between them (Gillam, 1976), and what is attached to them (Gillam, 1975). All of these investigations used lines with orientations of no more than $15^{\circ}$ from the horizontal, with their endpoints approximately aligned vertically, and, in all cases, rotation axis was vertical (see Figures 1A, 1B, and 1C).

In the following studies, we wished to see whether the grouping of two lines could be influenced by changing the orientation of the entire configuration

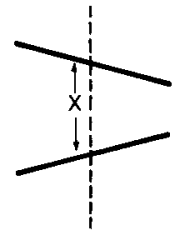

A

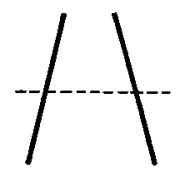

D

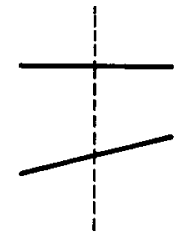

B

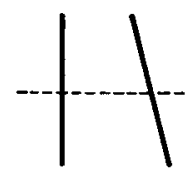

E
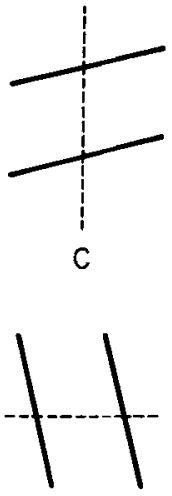

$\mathrm{F}$
Figure 1. Three of the stimulus patterns used in Experiments 1 and 2 , shown in two orientations. $A, B$, and $C$ show one orientation and $D, E$, and $F$ the other. The dotted line shows the axis of rotation. 
while relationships within the configuration, as well as its transformation over time, remained unaltered (Experiment 1). Such an effect would show that grouping depends not only on the relationships within the figure, but also on the relationship of the figure to external axes. If such effects were found, and they were, the next step (Experiment 2) was to see just which axes were important. That is, was degree of grouping related to the orientation of the figure relative to the subject's head/retina regardless of environmental axes on the one hand, or to its orientation relative to external environmental axes regardless of retinal axes, on the other? In other words, does grouping show orientation constancy or not?

\section{EXPERIMENT 1}

There were several reasons for thinking that pattern orientation might make a difference to the degree of grouping of two lines. Rotation of a horizontally symmetric pattern by $90^{\circ}$ would alter its axis of symmetry from horizontal to vertical. Previous patterns used, such as Figure 1A, always had a vertical axis of rotation and, if symmetric, their axis of symmetry was horizontal. Figure 1D shows the same pattern as it would look rotated $90^{\circ}$ with a horizontal axis of rotation and a vertical axis of symmetry. Symmetry around the vertical axis has been shown to be far more recognizable than symmetry about the horizontal (Julesz, 1971; Mach, 1906, 1959; Rock, 1973). Vertical symmetry might, therefore, as a recognizable form of redundancy, facilitate organization of the lines into a unit, which horizontal symmetry does not do (Gillam, 1972).

A second factor associated with rotating the configuration $90^{\circ}$ can be seen by comparing Figures $1 \mathrm{~A}$, $1 \mathrm{~B}$, and $1 \mathrm{C}$ with Figures $1 \mathrm{D}, 1 \mathrm{E}$, and $1 \mathrm{~F}$. The $90^{\circ}$ rotation of the patterns shown in Figures 1A, 1B, and $1 C$ to the positions shown in D, E, and F would place each of the two lines in a different hemisphere, assuming that the subject fixates the pattern centrally, as instructed. It is possible that projection into opposite hemispheres would weaken the connection between the lines so that their independence would be increased. However, previous research (Gillam, 1972) suggests that it is impossible to increase the independence of lines with very different orientations, such as Figure 1A, so any such hemisphere effect should be strongest for lines whose similarity in orientation produces more grouping to begin with, for example Figures 1B and IC (Gillam, 1972). Experiment 1 was designed to examine the effects on grouping of rotating the entire pattern and its axis of rotation by $90^{\circ}$ for a variety of patterns.

\section{Method}

The stimuli were generated on the face of a cathode ray tube (Hewlett-Packard 1310A X-Y display) using a minicomputer (Digital PDP-8/e with display control circuitry). Due to storage limitations, the computer was programmed to provide a static rendering of each pattern. The $X$ component of each point in the pattern was linearly multiplied by a sinusoidally varying signal before being sent to the display. This produced the equivalent of a parallel projection onto the frontal plane of a rotation in depth of the original pattern had it been a solid figure. The intensity of the stimulus varied slightly with time, increasing as the pattern "collapsed" because the total number of points in each stimulus was held constant. The pattern and its rotation axis could be shifted $90^{\circ}$ by interchanging $X$ and $Y$ inputs to the display unit. There were seven patterns, examples of which are shown in Figure 1 at the two orientations used. Six of the patterns, when presented in a vertical orientation, had a top line oriented $+15^{\circ}$ from the horizontal and bottom line orientations of $-30^{\circ},-15^{\circ}$, $-7.5^{\circ}, 0^{\circ},+7.5^{\circ}$, and $+15^{\circ}$ for patterns $1-6$, respectively. Pattern 3, with line orientations of $+15^{\circ}$ and $-15^{\circ}$, was symmetric. A second symmetric pattern was made with line orientations of $+30^{\circ}$ and $-30^{\circ}$ (pattern 7). Each pattern was shown twice, once with the line orientations close to horizontal (as shown for patterns 2, 4, and 6 in Figures 1A, 1B, and 1C) with a vertical axis of rotation, and once rotated $90^{\circ}$, i.e., with the line orientations close to vertical and with a horizontal axis of rotation (as shown for patterns 2, 4, and 6 in Figures 1D, 1E, and 1F). The entire 14 conditions (horizontal and vertical axes for each of seven patterns) were independently randomized for each subject. The visual angle subtended by the length marked $X$ in Figure 1 was $1^{\circ}$ at a viewing distance of $140 \mathrm{~cm}$. Speed of rotation was 5 revolutions/min.

Upon arrival, each subject was seated with his head in a chinrest and "tested" for spontaneous reporting of the illusion by viewing a shadow-casted rotating wire figure (Gillam, 1972, 1975). Each subject was instructed: "I want you to watch these moving lines and describe their movement to me. Be sure to look at the center of the figure." Only subjects spontaneously reporting that the two arms of the pattern appeared to rotate in opposite directions were allowed to complete the procedure (this criterion resulted in the rejection of approximately $10 \%$ of the subjects tested). Each subject was then provided with a switch which ran a counter and clock timer. The subject was instructed to press the switch and keep it down whenever the two lines appeared to be revolving in opposite directions. The time the switch was pressed down to indicate fragmentation of the pattern was cumulated over five revolutions $(60 \mathrm{sec})$ per pattern. Fragmentation time is assumed to be negatively related to grouping strength.

\section{Results}

The mean fragmentation times for 11 subjects for each pattern are shown in Table 1 . An analysis of variance showed a significant main effect for

Table 1

Mean Fragmentation Times for the Seven Figures of Experiment 1 for Vertical (V) and Horizontal (H) Axes of Rotation

\begin{tabular}{|c|c|c|c|c|c|c|c|c|c|c|c|c|c|}
\hline \multicolumn{2}{|c|}{1} & \multicolumn{2}{|c|}{2} & \multicolumn{2}{|c|}{3} & \multicolumn{2}{|c|}{4} & \multicolumn{2}{|c|}{5} & \multicolumn{2}{|c|}{6} & \multicolumn{2}{|c|}{7} \\
\hline $\mathrm{V}$ & $\mathrm{H}$ & $\mathrm{V}$ & $\mathrm{H}$ & $\mathrm{V}$ & $\mathbf{H}$ & $\mathrm{V}$ & $\mathrm{H}$ & $\mathrm{V}$ & $\mathrm{H}$ & $\mathrm{V}$ & $\mathrm{H}$ & $\mathrm{V}$ & $\mathrm{H}$ \\
\hline 30.5 & 23.0 & 26.3 & 17.4 & 30.1 & 19.8 & 28.2 & 21.3 & 17.6 & 18.3 & 12.4 & 11.1 & 22.1 & 10.3 \\
\hline
\end{tabular}

Note-Total rotation time for each figure was $60 \mathrm{sec}$. 
pattern $(\mathrm{F}=4.59, \mathrm{df}=6,60, \mathrm{p}<.01)$ and for axis/ orientation $(\mathrm{F}=5.6, \mathrm{df}=1,10, \mathrm{p}<.05)$. The interaction of pattern and axis was not significant. The horizontal axis of rotation combined with a line orientation close to vertical (examples of which are shown in Figures 1D, 1E, and 1F) showed less fragmentation (more tendency to be organized as a unit) than the vertical axis of rotation combined with a line-orientation close to horizontal (examples of which are shown in Figures 1A, 1B, and 1C).

\section{Discussion}

It is clear from the results that jointly changing the figure and rotation axis by $90^{\circ}$ did not have the effect predicted from hemispheric considerations. For most patterns, the condition in which opposite hemispheres were stimulated by the two lines resulted in a great deal more grouping of the lines, not less. The fact that a general effect of axis/orientation was obtained, with nonsymmetric as well as symmetric figures showing it, does not support the idea that the organizing power of vertical symmetry is the critical factor in producing greater grouping for the figures with a horizontal axis of rotation. Figure orientation seems to be important per se in determining perceptual grouping.

\section{EXPERIMENT 2}

The next step was to explore the nature of the orientation effect. Is it a postconstancy phenomenon depending upon the orientation of the pattern relative to the environment, or is it a preconstancy phenomenon depending upon the orientation of the pattern relative to the axis of the head/retina? Having the subject view rotating patterns with and without head tilt allows the environmental and head/ retina axes to be separated and their independent effects to be assessed.

\section{Method}

In this experiment, only those patterns shown in Figure 1 were used. The method of generating them and of measuring subjects' responses was the same as in Experiment 1, except that fragmentation time was cumulated over 10 revolutions per pattern, rather than 5. The 3 patterns at each of the two orientations were presented in random order to 10 subjects a total of four times-once per session for two sessions under each of two viewing conditions. The one presentation for each session was under normal viewing conditions (A) with the subject vertical and his head in a chinrest. For the other presentation (B), the subject viewed the patterns while lying on a mattress with his head horizontal, but in the same location as it was in the vertical viewing conditions. In the first session, for half the subjects the viewing conditions were in the order $\mathrm{AB}$ and for half they were in the order BA. Orders were reversed for the second session. Subjects were alternately assigned to viewing orders.

\section{Results}

The results are shown in Table 2. Even more clearly than in Experiment 1, there is a strong effect of axis, with less fragmentation for all patterns when they are oriented with their component lines close to vertical and rotated around a horizontal axis than when they are oriented with their component lines close to horizontal and rotated around a vertical axis. However, Table 2 shows an almost complete reversal of this effect when the body and head are tilted $90^{\circ}$. An analysis of variance of the data given in Table 2 shows a highly significant interaction of axis and orientation of subject $(F=40.38$, $\mathrm{df}=1,13, \mathrm{p}<.001){ }^{1}$. Since tilting the head and body $90^{\circ}$ reverses the relationship of the configuration to the retinal/head axis but does not alter its orientation relative to the environment, the strong effect of head tilt is a clear demonstration that the axis effect is tied to retinal and not to environmental coordinates.

\section{GENERAL DISCUSSION AND CONCLUSIONS}

The almost complete reversal of the axis effect as a result of head tilt shows that grouping, as measured by our method, has no orientation constancy. That is, head tilt is not compensated for either by an automatic correction resulting from proprioceptive/vestibular feedback or by a higher level process such as organization of the pattern relative to a top assigned in accordance with the environmental or gravitational vertical. We have noticed that knowledge of the stimulus conditions seems to have very little effect. A marked shift in fragmentation rate occurs even when the head is tilted during viewing of the rotating figure shown in Figure 1A.

With respect to its dependence on retinal axis, the orientation effect described here resembles orientation-contingent aftereffects and orientation differences in acuity (the so-called oblique effect) (Findlay \& Parker, 1972; Mitchell \& Blakemore, 1972), both of which depend on retinal orientation. It does not resemble tasks such as judgement of the vertical

Table 2

Mean Fragmentation Times for the Patterns Shown in Figure 1 for Vertical and Horizontal Axes of Rotation Combined with Vertical and Horizontal Orientations of the Subject

\begin{tabular}{lccc}
\hline Vertical Axis of Rotation & \multicolumn{3}{c}{ Horizontal Axis of Rotation } \\
\hline \multicolumn{4}{c}{ Subject Vertical } \\
A: & 23.45 & D: & 5.39 \\
B: & 19.02 & E: & 6.01 \\
C: & 31.45 & F: & 7.27 \\
Mean & 24.64 & Mean & 6.22 \\
& Subject Horizontal \\
A: & 2.89 & D: & \\
B: & 2.33 & E: & 25.50 \\
C: & 8.47 & F: & 12.36 \\
Mean & 4.56 & Mean & 19.85 \\
\hline
\end{tabular}

Note-Letters refer to Figure 1. 
in the dark, in which head and body tilt are compensated for or taken into account, or the perception of the orientation of objects in the environment, which depends very heavily on the relationship of object orientation to external reference points or axes. However, it is interesting that recognition is often disrupted to a considerable degree by retinal disorientation (Rock, 1973). This difficulty can be compensated for, to some extent, by active mental operations such as the assignment of a top to the figure, imagining rotation of the figure, and the use of certain critical features. These mental operations do not seem to be available to the grouping process. However, the profound changes we have shown in the organization of a figure when its retinal orientation is changed may well contribute significantly to the recognition difficulties that occur under conditions of retinal disorientation.

It is tempting to think of a task which depends on retinal orientation as independent of environmental influence. However, it is also possible that at least some retinal axis anisotropies are related to the way the environment typically projects onto the retina, but that at the early stages visual processing is relatively automatic and does not have a built-in or learned compensation for those infrequent cases in which retinal axes and environmental axes do not coincide.

Another criterion of grouping, originated by Beck (1967), is the ease or speed with which a set of elements can be perceptually segregated from another. Certain contrasting properties of the two sets of elements (such as orientation) result in much better grouping than other contrasting properties (such as position). Olsen and Attneave (1970) showed that horizontal lines amidst vertical lines, or vice versa, gave faster "grouping" than oblique line sets with a $90^{\circ}$ slope difference. They proceeded to ask the same question for their grouping criterion as concerned us in Experiment 2, namely, is the critical set of line orientations for optimal grouping determined by the retinal orientations or by the physical (gravitational) orientations of the lines? Their subjects were encouraged to use a gravitational reference system. The investigators found that physical orientation had a highly significant effect, although retinal orientation had some influence as well. They conclude that their results disconfirm the hypothesis that grouping occurs independently of constancy mechanisms, and attribute their result to the relationship between the stimulus elements and an implicit coordinate system. To what extent can their results be related to ours? Although the same term, taken from Gestalt psychology, has been used by both groups of investigators, the two grouping criteria may not be measuring the same perceptual process. It is true that elements which form a unit would be expected to stand out from those that are not part of the unit, but this may not be the only basis for segregation. Segregation does not imply organization among the segregated elements. It seems to have the function of directing attention (Beck, 1972), whereas the process we are tapping seems to have the function of defining objects and surfaces. Since we typically use only two lines, our task does not require segregation, in the sense of isolating certain elements for attention within a complex field. Segregation may be a prior process to organization, or it may serve an entirely different function under different stimulus conditions. There undoubtedly is a relationship between the two processes, but its nature has yet to be established. Therefore, our results should not be interpreted as conflicting with those of Olsen and Attneave.

In conclusion, we have shown that pattern orientation determines the degree to which two lines will form a perceptual unit and that this effect is dependent almost entirely on the orientation of the pattern relative to the retina/head and not relative to environmental or gravitational axes. It is argued that this places the grouping effect early in perceptual processing, but does not entirely rule out consideration of the way the environment normally projects onto the retina in explaining the effect.

\section{REFERENCES}

Beck, J. Perceptual grouping produced by line figures. Perception \& Psychophysics, 1967, 2, 491-495.

BECK, J. Similarity grouping and peripheral discriminability under uncertainty. American Journal of Psychology, 1972, 85, 1-19.

Findlay, J. M., \& Parker, D. M. An investigation of visual orientation-specific properties of acuity and adaptation. Perception, 1972, 1, 305-314.

Gillam, B. Perceived common rotary motion of ambiguous stimuli as a criterion of perceptual grouping. Perception \& Psychophysics, 1972, 11, 99-101.

Gillam, B. New evidence for closure in perception. Perception \& Psychophysics, 1975, 17, 521-524.

Gillam, B. Grouping of multiple ambiguous contours: Towards an understanding of surface perception. Perception, 1976, 5, 203-209.

Julesz, B. Foundations of cyclopean perception. Chicago: University of Chicago Press, 1971.

MACH, E. [The analysis of sensations.] (C. M. Williams, Trans.). New York: Dover Edition, 1959. (Originally published, 1906.)

Mitchell, D. E., \& Blakemore, C. The site of orientational constancy. Perception, 1972, 1, 315-320.

OzSĖN, R. K., \& ATtNeAve, F. What variables determine similarity grouping? American Journal of Psychology, 1970, 83, 1-21. Rock, I. Orientation and form. New York: Academic Press, 1973. Wallach, H., \& O'Connell, D. N. The kinetic depth effect. Journal of Experimental Psychology, 1953, 65, 205-217.

\section{NOTE}

1. The only other significant effects were the main effects for orientation $(\mathrm{F}=4.9$, df $=1,13, \mathrm{p}<.05)$ and the interaction of axis and orientation $(F=3.8$, $\mathrm{df}=2,13, \mathrm{p}<.05)$. The main effect for orientation cannot be accounted for by partial orientation constancy, since this would produce a regression to the ver- 
tical position response when the subject was horizontal, rather than a general increase in fragmentation when the subject was vertical, which is what we found. The most likely reason for this result was the greater comfort of subjects in the more natural vertical position, which might result in a tendency to press the switch more readily. The significant interaction of pattern with axis and orientation indicates that the effect of axis interacting with orientation was more powerful for some patterns than for others.

(Received for publication August 1, 1978; revision accepted May $28,1979$. 